\title{
Cellular-resolution in vivo tomography in turbid tissue through digital aberration correction
}

\author{
En Bo ${ }^{1+}$, Xin Ge ${ }^{1+}$, Yuemei Luo ${ }^{1}$, Xuan Wu${ }^{1}$, Si Chen ${ }^{1}$, Haitao Liang ${ }^{1}$, Shufen Chen ${ }^{1}$, Xiaojun Yu², Ping Shum',
} Jianhua $\mathrm{Mo}^{3}$, Nanguang Chen ${ }^{4}$ and Linbo Liu ${ }^{1,5^{*}}$

* Correspondence: liulinbo@ntu.edu.
sg
${ }^{\dagger}$ En Bo and Xin Ge contributed
equally to this work.
${ }^{1}$ School of Electrical and Electronic
Engineering, Nanyang
Technological University, 50
Nanyang Avenue, Singapore
639798, Republic of Singapore
${ }^{5}$ School of Chemical and Biomedical
Engineering, Nanyang
Technological University, 62
Nanyang Drive, Singapore 637459,
Republic of Singapore
Full list of author information is
available at the end of the article

Springer Open

\begin{abstract}
Noninvasive tomographic imaging of cellular processes in vivo may provide valuable cytological and histological information for disease diagnosis. However, such strategies are usually hampered by optical aberrations caused by the imaging system and tissue turbidity. State-of-the-art aberration correction methods require that the light signal be phase stable over the full-field data acquisition period, which is difficult to maintain during dynamic cellular processes in vivo. Here we show that any optical aberrations in the path length difference (OPD) domain can be corrected without the phase stability requirement based on maximum intensity assumption. Specifically, we demonstrate a novel optical tomographic technique, termed amplitude division aperture synthesis optical coherence tomography (ADAS-OCT), which corrects aberrations induced by turbid tissues by physical aperture synthesis and simultaneously data acquisition from sub-apertures. Even with just two subapertures, ADAS-OCT enabled in vivo visualization of red blood cells in human labial mucosa. We further demonstrated that adding sub-apertures could significantly scale up the aberration correction capability. This technology has the potential to impact a number of clinical areas where noninvasive examinations are preferred, such as blood count and cancers detection.
\end{abstract}

Keywords: Turbid tissue, Tomography, Aberration correction, Cellular resolution

\section{Introduction}

Imaging pathophysiological processes at the cellular level is critical for understanding and diagnosis of human diseases, which is routinely realized through cytological or histological examinations following biopsies in clinical settings. Because biopsy is associated with sampling errors and complications due to its invasive and destructive nature, it is not suitable for screening a large population or imaging over a large tissue area. Moreover, cytology and histology procedures are time-consuming which may result in delayed diagnosis. Tomographic imaging modalities are the mainstay of noninvasive diagnostic tools, including ultrasound imaging [1], computed tomography [2], magnetic resonance imaging [3] and positron emission tomography [4]. Although these

(c) The Author(s). 2020 Open Access This article is licensed under a Creative Commons Attribution 4.0 International License, which permits use, sharing, adaptation, distribution and reproduction in any medium or format, as long as you give appropriate credit to the original author(s) and the source, provide a link to the Creative Commons licence, and indicate if changes were made. The images or other third party material in this article are included in the article's Creative Commons licence, unless indicated otherwise in a credit line to the material. If material is not included in the article's Creative Commons licence and your intended use is not permitted by statutory regulation or exceeds the permitted use, you will need to obtain permission directly from the copyright holder. To view a copy of this licence, visit http://creativecommons.org/licenses/by/4.0/. 
existing tools have fundamentally changed clinical practice and basic research, cellularlevel cytological and histological information is still not available in tomograms in vivo due to the limited spatial resolution.

Optical tomography techniques are capable of achieving cellular-level spatial resolution in thin tissue sections or semi-transparent tissues [5-7]. However, imaging through thicker $(>300 \mu \mathrm{m})$ tissues in vivo is still highly challenging because the spatial resolution is degraded by optical aberrations, which can be roughly classified into system aberrations and sample-induced aberrations. System aberrations are generally inherent effects arising due to the focusing optics, such as defocus and inevitable imperfections in the optics used in endoscopic applications [8, 9]. Sample-induced aberrations in turbid tissues generally vary spatially and temporally. They must be corrected before clear images of cells can be obtained in vivo.

The straightforward way to correct these aberrations is using hardware-based adaptive optics (HAO), which measures the wavefront distortion and cancels it by modulating the incident light wavefront. With an HAO correction, a set of correction parameters is valid only over a sufficiently small volume and over a sufficiently short period of time during which the sample-induced aberrations are constant. Consequently, it is time consuming to screen a large tissue volume $[10,11]$. From a practical point of view, the system complexity and cost also impede its applications.

In OCT, optical aberrations result in not only the suboptimal coupling of signals at the detection pinhole but also interferometric wavefront distortions, which compromise both the coherence gating and the transverse focusing. Fortunately, the optical aberrations are recorded as the interferometric wavefront distortions that are detected optoelectronically, which opens up the possibility of correcting the aberrations digitally. Wide-field computational techniques, such as sub-aperture correlation [12, 13], computational adaptive optics [14-16], and model-based methods [17-19] have been developed to overcome the above-mentioned system complexity and speed issue with HAO. However, these techniques usually require high phase stability over the full-field acquisitions, which is challenging for imaging dynamic cellular processes in vivo, especially in endoscopic scenarios.

To overcome the limitation in phase stability, synthetic aperture OCT has been developed and demonstrated to be effective in digitally correcting the spherical aberration of the focusing optics [20]. However, it divides the aperture central-symmetrically by wavefront $[20]$ or sequentially by time $[21,22]$ so that it is not possible to correct the sample-induced aberrations. In this study, we report a novel technique, termed amplitude division aperture synthesis optical coherence tomography (ADAS-OCT), which corrects both the sample-induced aberrations and spherical aberrations without any phase stability requirement. We show that ADAS-OCT can deliver clear images of rapidly moving blood cells by restoring the spatial resolution degraded by the aberrations caused by the human mucosa in vivo.

\section{Methods}

\section{Working principle of aberration correction in ADAS-OCT}

In typical point-scanning confocal optics, when the wavefront of the optical beam is distorted by aberrations, destructive interference may occur at the detection pinhole, 
resulting in a broadened three-dimensional (3D) point-spread function (PSF) with a lower peak intensity compared with the diffraction-limited PSF (Fig. 1). Assuming the full aperture can be segmented into a number of sub-apertures, the aberrations in the optical path difference (OPD) domain can be digitally corrected if the OPD of light rays encircled in each sub-aperture can be recorded separately in the electrical signals. In spectral-domain OCT (SD-OCT), recording of the OPD is achieved by interference between the sample light with the reference light, governed by the following simplified formulation:

$$
I(k)=2 S(k) \int_{-\infty}^{\infty} \sqrt{I_{r}(k) I_{s}(k)} \cos (2 k z) d z
$$

where $I(k)$ is the spectral interference signal detected by the spectrometer, $S(k)$ is the spectral distribution of the light source and will be omitted in the following derivations, $I_{r}(k)$ and $I_{s}(k)$ are the electric field reflectivity from the reference arm and the sample arm at the depth of $z$, respectively. In the sample arm, one can synthesize the aperture of the objective lens by dividing the illumination beam by its amplitude and displace them evenly along the radial direction (Fig. 1). In the pupil plane, each beamlet creates a sub-aperture with distinct center spatial frequency, which defines both an illumination light path and a detection light path. The OPD of each distinct round-trip path can be shifted by a fixed length using an optical path encoder (Fig. 1) so that the corresponding interferometric signals do not overlap with each other in the OPD domain. In

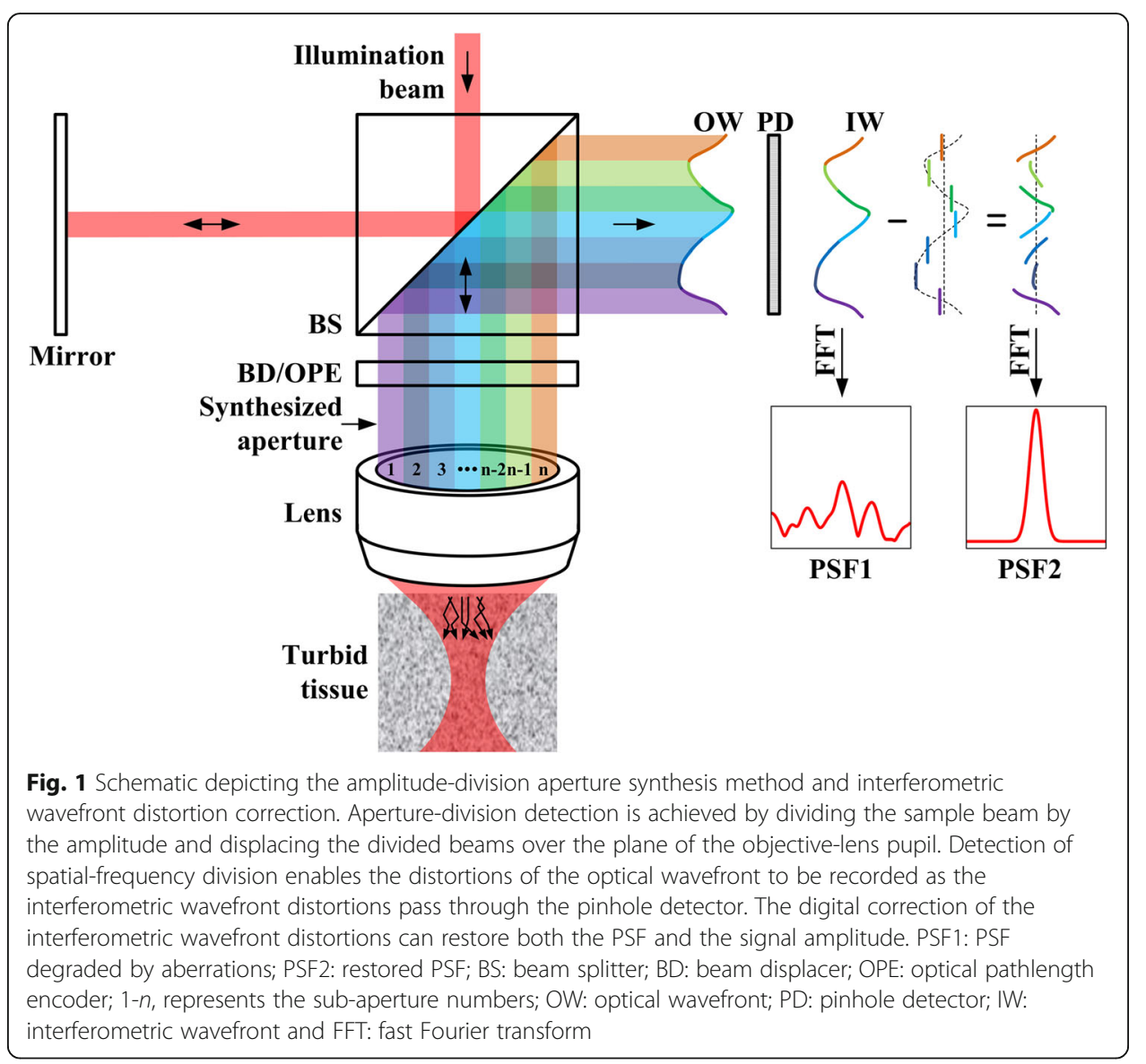


such an ADAS-OCT system, the $m$-th $(m=1,2, \ldots, 2 n-1$, represents the round-trip path number) depth-encoded cross-correlation term is given by the expression

$$
I_{m}(k)=\sqrt{I_{r}(k) I_{s}(k)}\left[\exp \left(i 2 k z_{m}\right)+C . C .\right]
$$

where C. C. stands for complex conjugate. Fourier transform $(\mathfrak{I})$ of these crosscorrelation terms give the standard SD-OCT images obtained through individual paths

$$
i_{S D-O C T, m}(z)=\mathfrak{I}\left[I_{m}(k)\right]
$$

Correction of aberrations in the OPD domain can be simply achieved by eliminating the phase delay $\alpha_{m}$ of $m$-th cross-correlation term $I_{m}$ with regard to $I_{1}$. Assuming that the aberration-free condition is met when the local intensity of coherently summed image reaches its maximum, the problem is equal to finding $\alpha_{m}^{c o h}$ which is the best estimate of $\alpha_{m}$ so that the local intensity $\max \left\{\left.i(z)\right|_{z \in\left[z^{\prime}, z^{\prime \prime}\right]}\right\}$ reaches its maximum, where $i($ $z)=\sum_{m=1}^{2 n-1} \mathfrak{3}\left[I_{m}(k) \cdot \exp \left(i \alpha_{m}\right)\right]$ is the coherent summation of the Fourier transform of the cross-correlation terms denoted by Eq. (2), $\left[z^{\prime}, z^{\prime \prime}\right]$ is the local depth range with $1 \leq$ $z^{\prime} \leq z^{\prime \prime} \leq N$, and $N$ is the length of Fourier transformation. Then the resultant aberration corrected A-line is given by

$$
i_{A D A S}(z)=\sum_{m=1}^{2 n-1} \mathfrak{I}\left[I_{m}(k) \cdot \exp \left(i \alpha_{m}^{c o h}\right)\right]
$$

While the interferometric signals from the local scatterer $\left(z^{\prime \prime}-z^{\prime}=10\right.$ coherence length) are coherently summed, those from the out-of-focus scatterers are suppressed due to OPD mismatches between light paths through different sub-apertures. Thus, this digital aberration correction mechanism can restore both the spatial resolution and the peak signal intensity degraded by aberrations. To produce the aberrationuncorrected control A-line, we incoherently summed the Fourier transform of the cross-correlation terms denoted in Eq. (2) so that $i(z)=\sum_{m=1}^{2 n-1}\left|\mathfrak{I}\left[I_{m}(k) \cdot \exp \left(i \alpha_{m}\right)\right]\right|$, and then found the optimal phase value for $\alpha_{m}$ so that global intensity $\max \left\{\mid i(z) \|_{z \in[1 \text {, }}\right.$ $\left.{ }_{N}\right\}$ reaches its maximum. Therefore, the aberration-uncorrected control A-line is given by,

$$
i_{\text {Control }}(z)=\sum_{m=1}^{2 n-1}\left|\mathfrak{I}\left[I_{m}(k) \cdot \exp \left(i \alpha_{m}^{i n c o h}\right)\right]\right|
$$

\section{ADAS-OCT imaging system}

We developed an ADAS-OCT system on an SD-OCT platform (Supplementary Fig. 1). Specifically, we created two sub-apertures using a birefringent beam displacer (Fig. 2a, Supplementary Fig. 2(a)-(c) and Supplementary Table 1), with which we obtained three cross-correlation terms with the aid of a quarter-wave plate: $I_{1}(k), I_{2}(k)$, and $I_{3}(k)$ are from the oo-path, the oe/eo-path, and the ee-path, respectively (Supplementary Fig. 2(d)). By adding a second birefringent beam displacer (Fig. 2b, Supplementary Fig. 2(e)(f) and Supplementary Table 2), we were able to create three sub-apertures and adjust the displacement between them, with which we could tailor the aberration correction performance. 


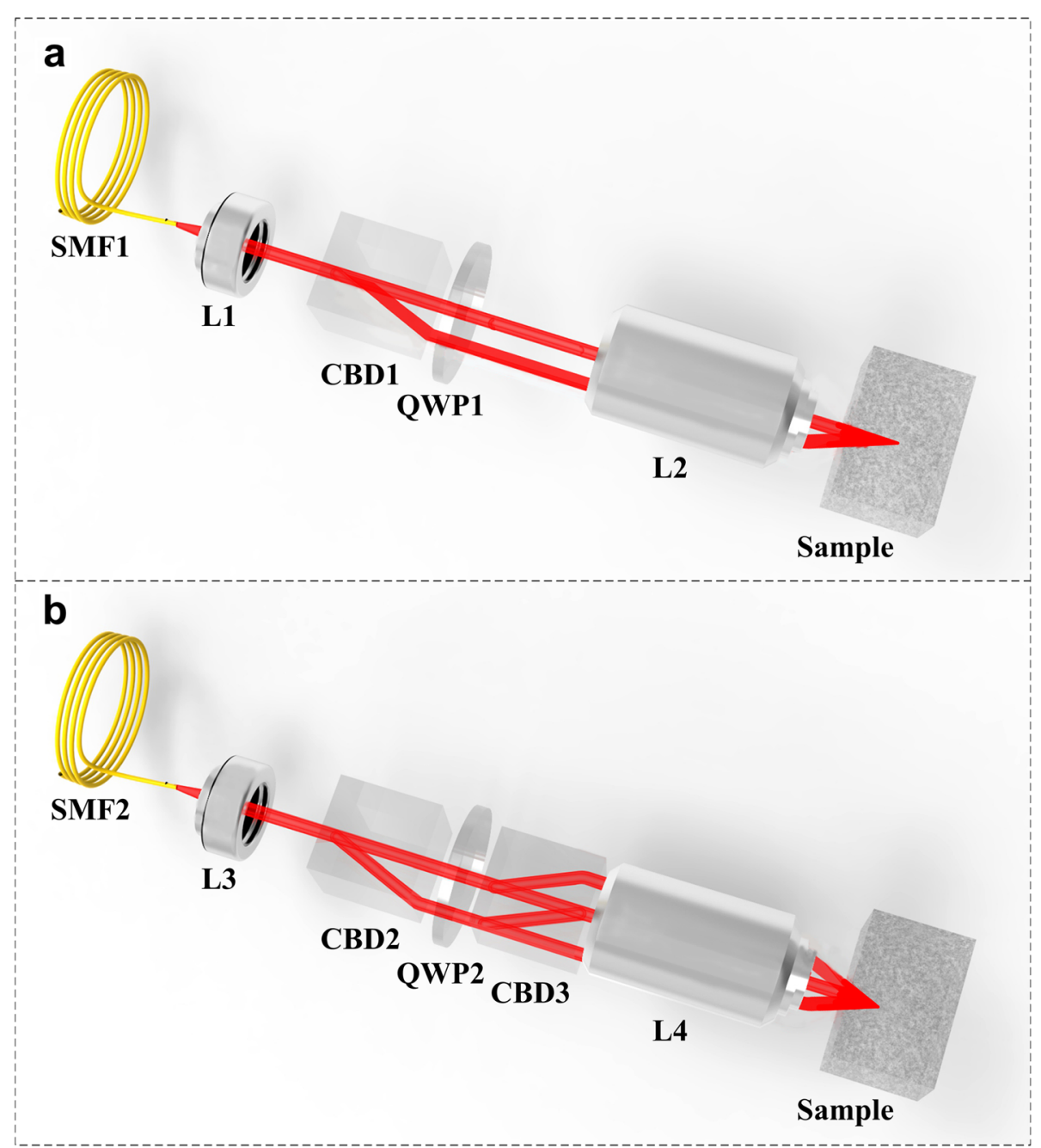

Fig. 2 Schematics of sample arms in ADAS-OCT with one (a) and two (b) calcite beam displacers demonstrating two and multiple distinctive apertures, respectively. SMF1-2, single mode fiber; L1-4, lens; CBD1-3, calcite beam displacer; QWP1-2, quarter wavelength plate

A light source with a spectral bandwidth of $180 \mathrm{~nm}$ centered at $800 \mathrm{~nm}$ was used to achieve an axial resolution of $1.50 \mu \mathrm{m}$ in water (refractive index $=1.33$ ). Meanwhile, the effective numerical aperture of the focusing optics of the SD-OCT platform was set to 0.13 (1\% power level) to achieve a transverse resolution of $2.75 \mu \mathrm{m}$ (full width at half maximum, FWHM) and a depth of focus of $30 \mu \mathrm{m}$ (confocal parameter). This spatial resolution allowed us to clearly visualize cellular structures under the aberration-free condition. Details of the system construction and signal transduction are provided in the Supplementary Notes 1 and 2.

\section{Results}

\section{Correction of sample-induced aberrations ex vivo}

To demonstrate the ability to correct sample-induced aberrations, we first imaged a simplified tissue phantom that consisted of a collection of polystyrene microparticles with a mean diameter of $6 \mu \mathrm{m}$ and that was uniformly suspended in agarose gel (See 
Supplementary Note 3 for details of phantom preparation). We focused the sample light to the upper half of the phantom through a chicken breast tissue with thickness of $289 \mu \mathrm{m}$ in air (Fig. 3a), resulting in broadened transverse and axial profiles of the particles in the control image (Fig. 3c, e, g) compared with an representative image obtained without the tissue on the phantom (Fig. 3b). In the aberration corrected image, both the transverse and axial profiles of the microparticles were significantly restored (Fig. $3 \mathrm{~d}, \mathrm{f}, \mathrm{h}$ ). For quantitative comparison, the FHWM values showed a mean improvement of $24.1 \%$ and $16.0 \%$ in the transverse and axial directions, respectively (Fig. $3 \mathrm{i}$ and j). In addition, a mean improvement in the peak intensity of $53.7 \%$ was obtained in favor of the signal-to-noise ratio (Fig. 3k). The image contrast and clarity of the chicken breast tissue were also significantly improved (boxes in Fig. 3c, d).

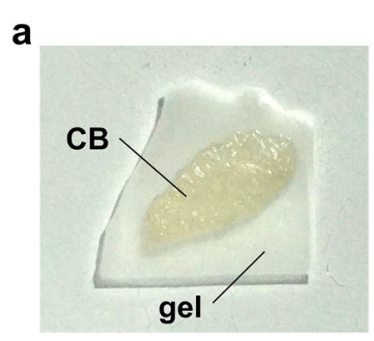

b

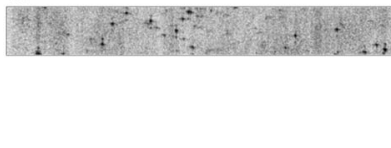

e
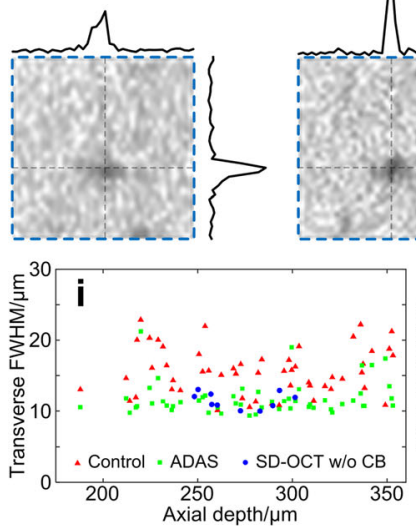
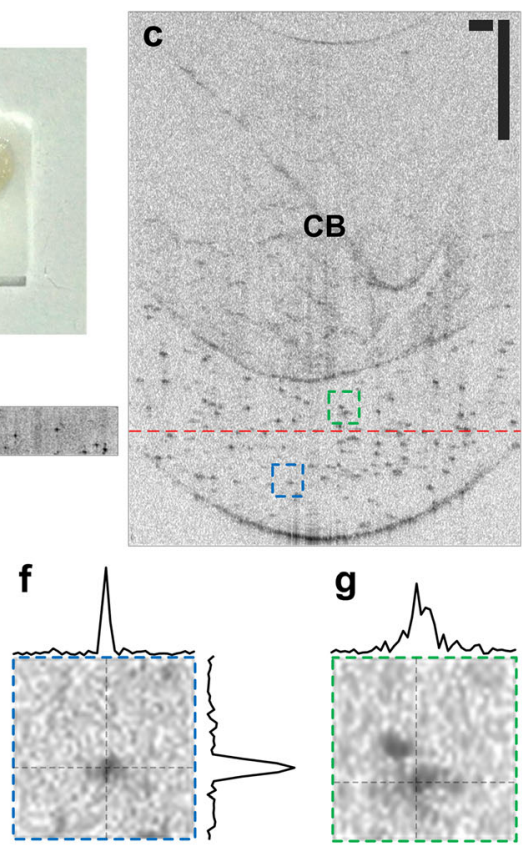

g

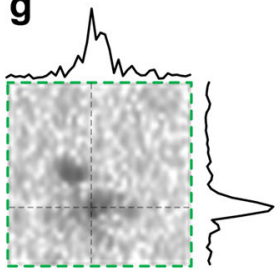

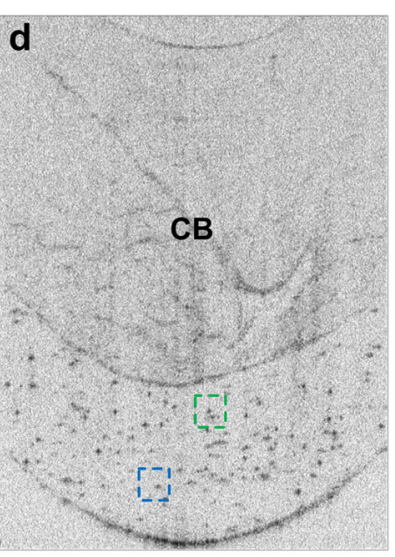

h
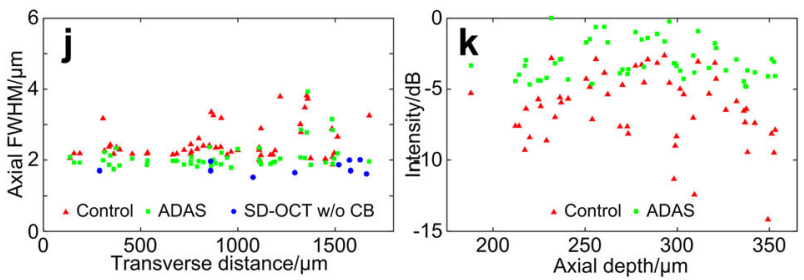

Fig. 3 Correction of sample-induced aberrations. a Photograph of the tissue phantom. CB: chicken breast. $\mathbf{b}$ An image of microparticles acquired without chicken breast tissue (SD-OCT w/o CB). c A representative control image (Control w/ CB). This was obtained using Eq. (5) with $\left[z^{\prime}, z^{\prime \prime}\right]$ being the full depth range. The red dashed line indicates the focal plane. $\mathbf{d}$ The corresponding aberration corrected image (ADAS w/ CB). e, $\mathbf{g}$ Six-fold magnified view of two insets in (c) show blurred microparticles. $\mathbf{f}, \mathbf{h}$ The corrected images corresponding to $(\mathbf{e})$ and $(\mathbf{g})$, respectively. The transverse and axial profiles are shown at the top and on the right of panels $(\mathbf{e})-(\mathbf{h})$, respectively. The profiles in $(\mathbf{e}) \&(\mathbf{g})$ are normalized to the peak values in (f) and (h), respectively. $\mathbf{i}$ and $\mathbf{j}$ The FWHM of the transverse and axial profiles are restored in the ADAS-OCT image (ADAS w/ CB, green rectangle) with respect to the control image (Control w/ CB, red triangle) and the SDOCT image (SD-OCT w/o CB, blue circle). $\mathbf{k}$ Signal intensity is also restored in the ADAS-OCT image (green rectangle) with respect to the control image (red triangle). Here, "Control" and "ADAS" represent the aberration-uncorrected and aberration-corrected images with chicken breast given by Eqs. (4) and (5) respectively, while "SD-OCT" is the conventional OCT image of the particle phantom only without aperturedivision. Transverse and vertical scale bars: $100 \mu \mathrm{m}$ 


\section{Correction of sample-induced aberration in vivo}

RBCs are ideal image phantoms due to their characteristic axial scattering profiles and spatial sparsity in blood vessels. According to our previous observations in zebra fish larvae, a typical OCT image of an RBC consists of two coherence-length limited reflection signals from the upper and lower boundaries of the cell's biconcave disk [23]. Under the aberration-free condition, RBCs should be easily identified in OCT images since the platelets and white blood cells are much fewer and present very different scattering characteristics $[24,25]$. We imaged the labial mucosa of human volunteers with the focal region aligned to the lamina propria (See Supplementary Note 3 for details of sample preparation). The optical thickness (refractive index $=1.33$ ) was approximately $300 \mu \mathrm{m}$ from the surface of the stratified squamous epithelium to the RBCs in focus. In a representative aberration-corrected ADAS-OCT image (Fig. 4b, d), we could clearly identify six pairs of reflectance signals, presumably six RBCs (Fig. 4e). In contrast, in the corresponding control image (Fig. 4a, c), two reflective surfaces of RBCs numbered 4-6 are difficult to discern due to aberrations. It is obvious that aberration correction significantly enhanced the peak signal intensity of RBCs as compared with the control image (Fig. 4f), which contributed to the visibility of RBCs. In one of the other images, we could recover signals of RBCs even when one or both reflection signals were lost due to aberrations (Supplementary Figs. S3 and S4).

\section{Scalable imaging performance}

The performance of the ADAS-OCT technique can be enhanced by scaling up the number of sub-apertures and increasing the displacement between them. Using the dual beam displacer setup (Fig. 2b and Supplementary Fig. 2(e)-(f)), we obtained three equally displaced sub-apertures: $o o$, ee, and oe/eo. The displacement between the centers of the sub-apertures $\Delta x$ was adjusted in terms of $\mathrm{R}$ by rotating the second displacer ( $R=1.3 \mathrm{~mm}$ is the radius of the illumination beam measured at the $1 \%$ power level).

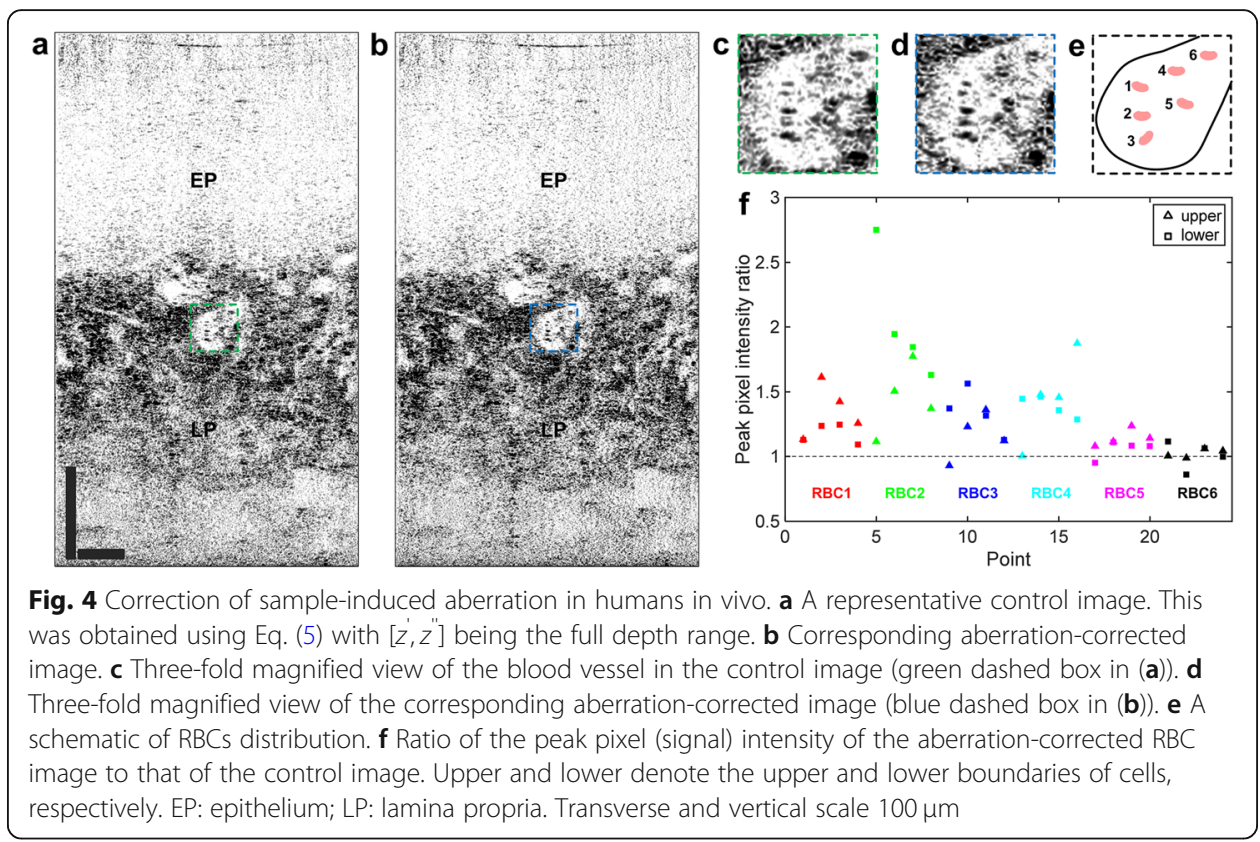


This scalability was demonstrated by correcting defocus, which is the simplest aberration but represents a general problem in microscopy and endoscopy. As before, we used the tissue phantom consisting of 6 - $\mu \mathrm{m}$-diameter microparticles suspended in agarose gel. At a sample location $250 \mu \mathrm{m}$ above the focal plane, the mean transverse FWHM of the particles was degraded to $24.1 \mu \mathrm{m}, 25.6 \mu \mathrm{m}$, and $27.1 \mu \mathrm{m}$ in the control images (Fig. 5a-c and red line in $\mathrm{j}-1$ ).

For the group of two sub-apertures ( $o o$ and $e e$ ), the transverse FWHM was found to improve by $9.1 \%$ (for displacement between sub-apertures, $\Delta x=0.2 \mathrm{R}$ ), $25.8 \%$ (for $\Delta x=1 \mathrm{R}$ ) and $57.2 \%$ (for $\Delta x=1.8 \mathrm{R}$ ) (Fig. $5 \mathrm{~d}$-f and green line in $\mathrm{j}-1$ ). Larger displacement results in better correction also held true in the group of three sub-apertures (oo, ee, and oe/eo) (Fig. $5 \mathrm{~g}-\mathrm{i}$ and blue line in $\mathrm{j}-1)$. In the largest displacement of $1.8 \mathrm{R}$ between the oo sub-aperture and the ee sub-aperture, the mean transverse FWHM was $9.2 \mu \mathrm{m}$ with three subapertures, which was $69.4 \%$ better than that of the control image data (Fig. 5l), while the mean transverse FWHM was $11.6 \mu \mathrm{m}$ with two sub-apertures. It is suggested that the number of sub-apertures is related to the improvement of the transverse FWHM.

Wavefront distortions between sub-apertures in the path delay domain are digitally corrected by matching the optical path delay of light rays encircled in sub-apertures. By scaling up the sub-apertures number, wavefront distortions (such as IW in Fig. 1) in the aperture plane could be sampled finer to achieve a more accurate approximation. If we could have an infinitely large number of sub-apertures, we will be able to compensate for higher-order

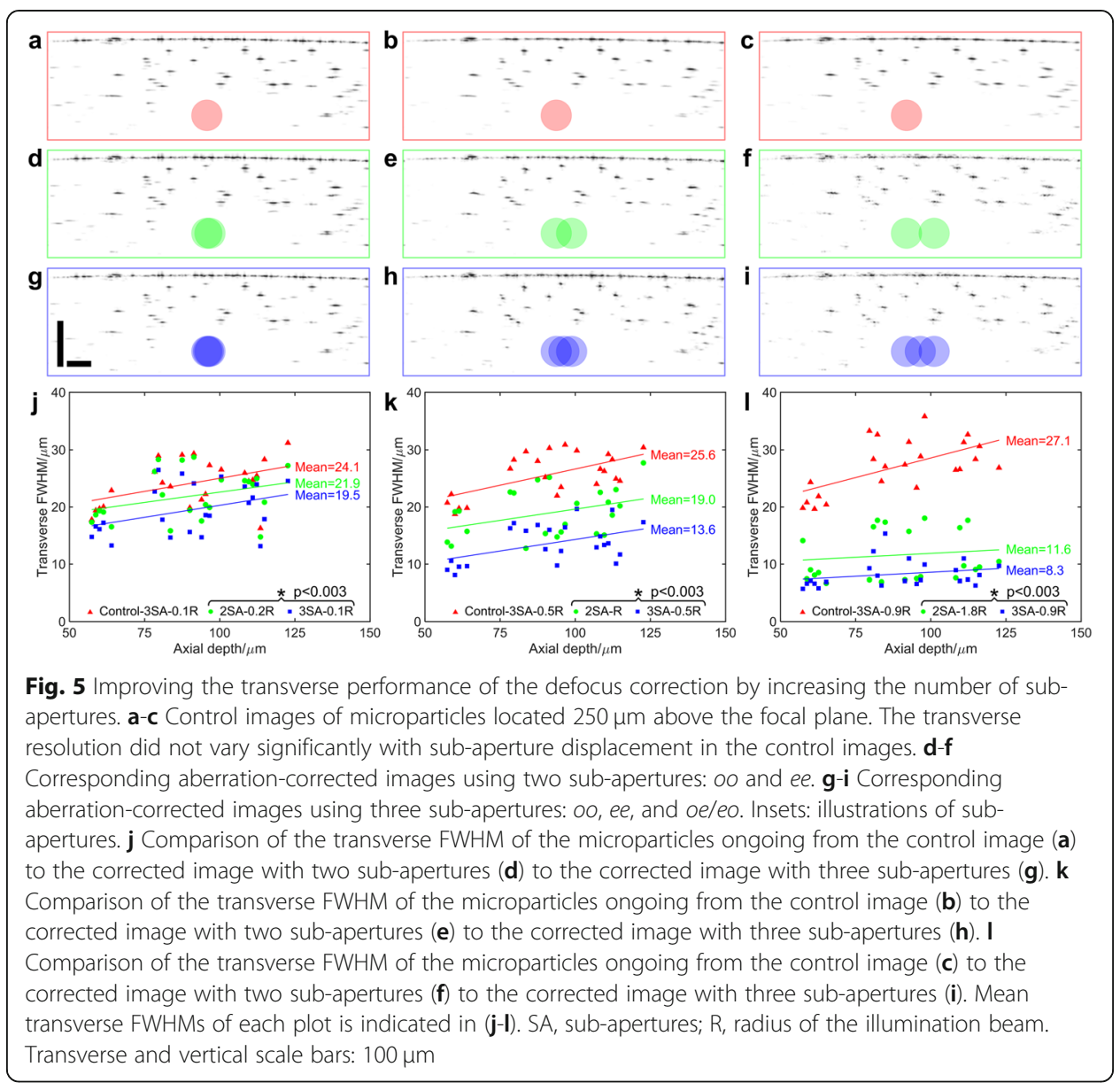


wavefront distortions, but it will never achieve the same diffraction limited transverse resolution as a standard focusing system with an aperture being all the sub-apertures combined since ADAS refocusing is one dimensional (depth) instead of three dimensional.

\section{Discussion}

ADAS-OCT is based on the same depth-priority point-scanning mechanism as the most of current endoscopic and intracoronary OCT systems, so that it can be potentially developed into an endoscopic technology that performs high-speed circumferential and helical scanning. In contrast, to maintain phase stability high enough to guarantee coherent aperture synthesis, existing computational-based aberration-correction methods have to rely on special scanning mechanisms such as line-field scanning or time-domain lateral priority scanning [14-16], which are not suitable for endoscopic applications in tubular organs. An ADAS-OCT endoscopic probe could be realized in practice by incorporating a beam displacer and a polarizer into a classical fiber-optic design [26]. Beam splitter (BS) is an alternative beam displacer (Supplementary Fig. 5, see Supplementary Note 4 for details of beam division). However, it is not easy to fabricate this two-surface coating BS with a diameter of 1-2 $\mathrm{mm}$ in an ADAS-OCT endoscopic probe. Considering the design, the manufacture, and the test of beam displacer, birefringent crystal should be a better choice. Using a calcite beam displacer, the two-beam separation angle could be $2.2^{\circ}$ with a probe diameter of $2 \mathrm{~mm}$ and a rigid length of $21.2 \mathrm{~mm}$, based on optical simulation results using Zemax software (Zemax, LLC, Kirkland, Washington, USA) (Supplementary Fig. 6). This probe diameter should be compatible with the instrument channel of a standard video endoscope [27] or in a tethered capsule endomicroscope [26, 28].

Once developed, such an ADAS-OCT endoscope may enable tomographic diagnosis of mucosal abnormalities at the cellular basis in internal organs. Potential clinical benefits may include the early detection of intraepithelial neoplasia and metaplasia in the gastrointestinal tract. Such early detection can be missed by standard video endoscopy and random biopsy procedures if the lesions are focally and heterogeneously distributed over a large mucosal area, leading to a lack of morphological features at the mucosal surface. ADAS-OCT could potentially improve screening and diagnostic outcomes by capturing the cellular hallmarks of the precancerous lesions invisible to standard-ofcare tools, such as goblet cells in Barratt's esophagus and specialized intestinal metaplasia in the stomach.

Looking towards the goal of tomographic diagnosis at the cellular level, future efforts will focus on the development of a clinically viable endomicroscopic ADAS-OCT system. The diameter and rigid length of the probe may be further reduced by using materials with larger birefringence, such as yttrium orthovanadate (YVO4). Space constraints could be further mitigated by employing a tethered capsule that can accommodate largerdiameter optics. The ranging depth of the spectrometer (Supplementary Fig. 7) could be extended through the use of spectral combination [23], linearization [29], and circular interferometric ranging [30].

\section{Conclusion}

ADAS-OCT offers a relatively simple route to cellular-level resolution tomography through turbid tissues in vivo. Our approach avoids the phase stability issue encountered by existing computational-based aberration-correction methods. Current computational 
formulations require full-field data acquisition, during which motion-induced phase instability is inevitable. ADAS-OCT is inherently insensitive to motion-induced phase variations or spatially variant wavefront distortions because it collects scattered signals from all sub-apertures simultaneously. Therefore, to the best of our knowledge ADAS-OCT is the first method that can correct tissue induced aberrations during a rapid cellular process, such as blood flow.

This technical advance may enable noninvasive blood count, which will potentially address many challenges in diagnosis, management, and treatment of diseases. Neonatal blood sampling represents one type of clinical challenges where blood volume and accessible veins are limited for needle puncture, and capillary skin puncture may be associated with increased risk of infection, injury, pain, and maternal anxiety [31, 32]. While, blood count monitoring during chemotherapy represents another type of challenges where the venipuncture-based blood test may lead to complications, and delays and/or dose reductions of subsequent courses of chemotherapy [33, 34]. A non-invasive blood count device enabled by ADAS-OCT could potentially reduce the number of blood samples from newborns and allow rapid tailoring the dosage and improve the life quality of patients undergoing chemotherapy.

\section{Supplementary information}

Supplementary information accompanies this paper at https://doi.org/10.1186/s43074-020-00009-7.

Additional file 1: Supplementary Figure 1. Schematic of the ADAS-OCT system. L1-5: optical lenses; L6: camera lens; G: transmission diffraction grating; NPBS: non-polarizing beam splitter; CBD: calcite beam displacer; QWP: quarter-wave plate; GS: two-dimensional galvo scanner; LP: linear polarizer; DC: dispersion compensator; NDF: neutral density filter; M: mirror; IMAQ: image acquisition system; PC: personal computer; $\mathrm{AO}$ : analog output; $\mathrm{O}^{-}$and $e^{-}$, the ordinary beam and the extraordinary beam. Supplementary Figure 2. Schematic of the sample arm optics of ADAS-OCT. (a) Sample arm optics with a beam displacer and a quarter-wave plate, where the red beam is the combination of the $O$ - and e-beams, the green beam is the e-beam, and the blue beam is the o-beam. SMF: singlemode fiber; L1: collimating lens; CBD: calcite beam displacer; OA: optical axis of the CBD (in plane of paper); QWP: quarter-wave plate; L2: focusing lens; 0 : ordinary beam; $e$ : extraordinary beam; $\Delta z$, the single-trip OPD caused by the CBD between the $o$ - and e-beams. (b) Illustration of birefringent beam displacement. PS: principle section; $d$ : length of the CBD; $\Delta x$ : separated distance of the $o$ - and e-beams perpendicular to the direction of propagation; $\varphi$ : angle of separation of the e-beam in the $\mathrm{CBD} ; \varphi$ : angle between the slow axis and $\mathrm{OA}$ of the CBD; $\theta$ : angle between the fast axis and $\mathrm{OA}$ of the $\mathrm{CBD} ; E_{/ /}$and $E_{\perp}$, transverse and vertical electric field, respectively. (c) Each of the displaced beams was circularly polarized on the round trip to maximize the coupling back to the fiber pinhole. (d) The four round-trip optical paths taken by the light. (e) The number of sub-apertures can be scaled up through the use of cascading beam displacers, where the yellow beam is the combination of the oo- and ee-beams. ( $f$ ) Rotation of CBD2 generates maximum aperture separation. Supplementary Figure 3. One of the other representative images of RBCs in vivo. (a-c) $m$-th $(m=1-3)$ uncorrected images (a-c) from the oo-path, oe/eo-path, and the ee-path, respectively. (d) The control image ([z, $\left.z^{\prime \prime}\right]$ being the full depth range). In all the uncorrected images and the aberration-corrected image, the three RBCs (green arrows) were not clearly identifiable due to the lack of characteristic 'two reflection signals'. (e) Aberration corrected image, where the characteristic 'two reflection signals' are clearly visible. Transverse and vertical scale bars: $100 \mu \mathrm{m}$. Supplementary Figure 4. Another one of the other representative images of RBCs in vivo. (a-c) $m$-th $(m=1-3)$ uncorrected images $(a-c)$ from the oo-path, oe/eo-path, and the ee-path, respectively. (d) The control image ([z, $\left.z^{\prime \prime}\right]$ being the full depth range). In all the uncorrected images and the aberration-corrected image, the three RBCs (green arrows) were not clearly identifiable due to the lack of characteristic 'two reflection signals'. (e) Aberration corrected image, where the characteristic 'two reflection signals' are clearly visible. Transverse and vertical scale bars: $100 \mu \mathrm{m}$. Supplementary Figure 5. Principle of Fourier multiple aperture synthesis based on the beam splitter (BS). (a) Two optical beams generated by the BS. (b) Illumination apertures and detection apertures of two optical beams indicated in (a). (c) Four round-trip light paths based on illumination apertures and detection apertures, respectively. SMF, single mode fiber, BS: beam splitter with reflective films coated on the first and second surfaces; S1-2, the first and second coated surfaces; L1, collimation lens; L2, objective lens; A1-2, two optical apertures generated by the BS. $\Delta d$ is the transverse distance between adjacent illumination beams. Supplementary Figure 6. Design of the ADAS-OCT probe using Zemax optical simulation software. 1: Glass spacer (BK7, diameter $2 \mathrm{~mm}$, length $6.95 \pm 0.05 \mathrm{~mm}$ ). 2: GRIN lens 1 (IFRL-200-005-50, length $1.23 \pm 0.005$ ). 3: Calcite (diameter $2 \mathrm{~mm}, 7.4 \pm 0.01 \mathrm{~mm}$ ). 4: Polarization analyzer (diameter $2 \mathrm{~mm}, 1.7 \pm 0.05 \mathrm{~mm}$ ). 5 : GRIN lens 2 (IFRL-200-005-50, length $1.23 \pm 0.005$ mm). 6: Prism (BK7, leg $0.65 \mathrm{~mm}$ ). $\varphi$ : Separation angle between the $o$-beam (blue) and the e-beam (red), with value 2.2 $2^{\circ}$ The designed working distance $L$ is $1.52 \pm 0.02 \mathrm{~mm}$. Supplementary Figure 7. Measured axial PSFs (on a linear scale). The signal intensity drops by $6 \mathrm{~dB}$ at a depth of $1.87 \mathrm{~mm}$. Supplementary Table 1. Design parameters of the calcite beam displacer in Fig. 2a and Supplementary 
Fig. 2(a). Supplementary Table 2. Design parameters of the calcite beam displacers in Fig. $2 \mathrm{~b}$ and Supplementary Fig. 2 (e)-(f). Supplementary Note 1. ADAS-OCT imaging system. Supplementary Note 2. Amplitude division and round-trip beam paths. Supplementary Note 3. Sample preparation and visualization. Supplementary Note 4. Beam splitter acts as a beam displacer.

\section{Acknowledgements}

Not applicable.

\section{Authors' contributions}

E.B., X.G., N.C. and L.L. conceived the study. E.B. and X.G. developed ADAS-OCT method, conducted the imaging studies and participated in writing the manuscript. Y.L., X. W, S.C., H.L., S.C., X.Y., J.M., and P.S. contributed to the imaging studies. L.L. supervised the overall project and participated in writing the manuscript. The authors read and approved the final manuscript.

\section{Funding}

This research was supported by National Research Foundation Singapore under its Competitive Research Program (NRF-CRP13-2014-05), Ministry of Education Singapore under its Academic Research Fund Tier 1 (2018-T1-001-144), and Agency for Science, Technology and Research (A*STAR) under its Industrial Alignment Fund (Pre-positioning) (H17/01/a0/008).

\section{Availability of data and materials}

Requests for materials should be addressed to liulinbo@ntu.edu.sg (L.L.).

\section{Competing interests}

The authors declare that they have no competing interests.

\section{Author details}

'School of Electrical and Electronic Engineering, Nanyang Technological University, 50 Nanyang Avenue, Singapore 639798, Republic of Singapore. ${ }^{2}$ School of Automation, Northwestern Polytechnical University, Xi'an 710072, Shaanxi, China. ${ }^{3}$ School of Electronics and Information Engineering, Soochow University, Shizi Street 1, Suzhou 215006, China. ${ }^{4}$ Department of Biomedical Engineering, National University of Singapore, Singapore 117583, Republic of Singapore. ${ }^{5}$ School of Chemical and Biomedical Engineering, Nanyang Technological University, 62 Nanyang Drive, Singapore 637459, Republic of Singapore.

Received: 26 July 2019 Accepted: 20 February 2020

Published online: 12 March 2020

\section{References}

1. Fenster A, Downey DB, Cardinal HN. Three-dimensional ultrasound imaging. Phys Med Biol. 2001;46(5):R67.

2. Hsieh J. Computed tomography: principles, design, artifacts, and recent advances. SPIE press, 2003.

3. Rooney W. MRI: from picture to proton. Health Phys. 2003;85(4):504-5.

4. Bailey D L, Maisey M N, Townsend D W, et al. Positron emission tomography. London: Springer; 2005.

5. Ntziachristos V. Going deeper than microscopy: the optical imaging frontier in biology. Nat Methods. 2010;7(8):603.

6. Sharpe J, Ahlgren U, Perry P, et al. Optical projection tomography as a tool for 3D microscopy and gene expression studies. Science. 2002;296(5567):541-5.

7. Power RM, Huisken J. A guide to light-sheet fluorescence microscopy for multiscale imaging. Nat Methods. 2017;14(4): 360.

8. Hermann B, Fernández E, Unterhuber A, et al. Adaptive-optics ultrahigh-resolution optical coherence tomography. Opt Lett. 2004:29(18):2142-4.

9. Benalcazar WA, Jung W, Boppart SA. Aberration characterization for the optimal design of high-resolution endoscopic optical coherence tomography catheters. Opt Lett. 2012;37(6):1100-2.

10. Zawadzki RJ, Jones SM, Olivier SS, et al. Adaptive-optics optical coherence tomography for high-resolution and highspeed 3D retinal in vivo imaging. Opt Express. 2005;13(21):8532-46.

11. Jang J, Lim J, Yu H, et al. Complex wavefront shaping for optimal depth-selective focusing in optical coherence tomography. Opt Express. 2013;21 (3):2890-902.

12. Ginner L, Kumar A, Fechtig D, et al. Noniterative digital aberration correction for cellular resolution retinal optical coherence tomography in vivo. Optica. 2017;4(8):924-31.

13. Hillmann D, Spahr H, Hain C, et al. Aberration-free volumetric high-speed imaging of in vivo retina. Sci Rep. 2016;6: 35209 .

14. Adie SG, Graf BW, Ahmad A, et al. Computational adaptive optics for broadband optical interferometric tomography of biological tissue. Proc Natl Acad Sci. 2012;109(19):7175-80.

15. Shemonski ND, South FA, Liu Y-Z, et al. Computational high-resolution optical imaging of the living human retina. Nat Photonics. 2015;9(7):440.

16. Ahmad A, Shemonski ND, Adie SG, et al. Real-time in vivo computed optical interferometric tomography. Nat Photonics. 2013;7(6):444.

17. Yasuno Y, Sugisaka J-i, Sando Y, et al. Non-iterative numerical method for laterally superresolving Fourier domain optical coherence tomography. Opt Express. 2006;14(3):1006-20.

18. Kumar A, Drexler W, Leitgeb RA. Numerical focusing methods for full field OCT: a comparison based on a common signal model. Opt Express. 2014;22(13):16061-78.

19. Ralston TS, Marks DL, Carney PS, et al. Interferometric synthetic aperture microscopy. Nat Phys. 2007;3(2):129. 
20. Mo J, de Groot M, de Boer JF. Focus-extension by depth-encoded synthetic aperture in optical coherence tomography. Opt Express. 2013;21(8):10048-61.

21. Bo E, Luo Y, Chen S, et al. Depth-of-focus extension in optical coherence tomography via multiple aperture synthesis. Optica. 2017:4(7):701-6.

22. Bo E, Ge X, Wang L, et al. Multiple aperture synthetic optical coherence tomography for biological tissue imaging. Opt Express. 2018;26(2):772-80.

23. Cui D, Liu X, Zhang J, et al. Dual spectrometer system with spectral compounding for 1- $\mathrm{um}$ optical coherence tomography in vivo. Opt Lett. 2014;39(23):6727-30.

24. Liu L, Gardecki JA, Nadkarni SK, et al. Imaging the subcellular structure of human coronary atherosclerosis using microoptical coherence tomography. Nat Med. 2011;17(8):1010.

25. Chu KK, Kusek ME, Liu L, et al. Illuminating dynamic neutrophil trans-epithelial migration with micro-optical coherence tomography. Sci Rep. 2017;7:45789.

26. Gora MJ, Sauk JS, Carruth RW, et al. Tethered capsule endomicroscopy enables less invasive imaging of gastrointestinal tract microstructure. Nat Med. 2013;19(2):238-40.

27. Gora MJ, Suter MJ, Tearney GJ, et al. Endoscopic optical coherence tomography: technologies and clinical applications, Biomed Opt Express. 2017;8(5):2405-44.

28. Liang K, Traverso G, Lee H-C, et al. Ultrahigh speed en face OCT capsule for endoscopic imaging. Biomed Opt Express. 2015;6(4):1146-63.

29. Lan G, Li G. Design of a k-space spectrometer for ultra-broad waveband spectral domain optical coherence tomography. Sci Rep. 2017;7:42353.

30. Siddiqui M, Nam AS, Tozburun $\mathrm{S}$, et al. High-speed optical coherence tomography by circular interferometric ranging. Nat Photonics. 2018;12(2):111

31. Meites S. Skin-puncture and blood-collecting technique for infants: update and problems. Clin Chem. 1988;34(9):1890-4.

32. Shah VS, Taddio A, Bennett S, et al. Neonatal pain response to heel stick vs venepuncture for routine blood sampling. Arch Dis Child Fetal Neonatal Ed. 1997;77(2):F143-4.

33. Chirivella I, Bermejo B, Insa A, et al. Impact of chemotherapy dose-related factors on survival in breast cancer patients treated with adjuvant anthracycline-based chemotherapy. J Clin Oncol. 2006;24(18_suppl):668.

34. Clamp AR, Ryder WDJ, Bhattacharya S, et al. Patterns of mortality after prolonged follow-up of a randomised controlled trial using granulocyte colony-stimulating factor to maintain chemotherapy dose intensity in non-Hodgkin's lymphoma. Br J Cancer. 2008;99(2):253.

\section{Publisher's Note}

Springer Nature remains neutral with regard to jurisdictional claims in published maps and institutional affiliations.

\section{Submit your manuscript to a SpringerOpen ${ }^{\circ}$ journal and benefit from:}

- Convenient online submission

- Rigorous peer review

- Open access: articles freely available online

High visibility within the field

- Retaining the copyright to your article

Submit your next manuscript at $\boldsymbol{\nabla}$ springeropen.com 